Portland State University

PDXScholar

\title{
Effects of Ocean Thermocline Variability on Noncoherent Underwater Acoustic Communications
}

\author{
Martin Siderius \\ Portland State University, siderius@pdx.edu \\ Michael B. Porter \\ HLS Research Corporation \\ Paul Hursky \\ HLS Research Corporation \\ Vincent McDonald \\ HLS Research Corporation
}

Follow this and additional works at: https://pdxscholar.library.pdx.edu/ece_fac

Part of the Electrical and Computer Engineering Commons Let us know how access to this document benefits you.

\section{Citation Details}

Siderius, M., Porter, M. B., Hursky, P., \& McDonald, V. (2007). Effects of ocean thermocline variability on noncoherent underwater acoustic communications. Journal of The Acoustical Society of America, 121(4), 1895-1908.

This Article is brought to you for free and open access. It has been accepted for inclusion in Electrical and Computer Engineering Faculty Publications and Presentations by an authorized administrator of PDXScholar. Please contact us if we can make this document more accessible: pdxscholar@pdx.edu. 


\title{
Effects of ocean thermocline variability on noncoherent underwater acoustic communications
}

\author{
Martin Siderius, ${ }^{\text {a) }}$ Michael B. Porter, Paul Hursky, Vincent McDonald, and \\ the KauaiEx Group \\ HLS Research Corporation, 12730 High Bluff Drive, San Diego, California 92130
}

(Received 16 December 2005; revised 14 December 2006; accepted 30 December 2006)

\begin{abstract}
The performance of acoustic modems in the ocean is strongly affected by the ocean environment. A storm can drive up the ambient noise levels, eliminate a thermocline by wind mixing, and whip up violent waves and thereby break up the acoustic mirror formed by the ocean surface. The combined effects of these and other processes on modem performance are not well understood. The authors have been conducting experiments to study these environmental effects on various modulation schemes. Here the focus is on the role of the thermocline on a widely used modulation scheme (frequency-shift keying). Using data from a recent experiment conducted in 100-m-deep water off the coast of Kauai, HI, frequency-shift-key modulation performance is shown to be strongly affected by diurnal cycles in the thermocline. There is dramatic variation in performance (measured by bit error rates) between receivers in the surface duct and receivers in the thermocline. To interpret the performance variations in a quantitative way, a precise metric is introduced based on a signal-to-interference-noise ratio that encompasses both the ambient noise and intersymbol interference. Further, it will be shown that differences in the fading statistics for receivers in and out of the thermocline explain the differences in modem performance. (c) 2007 Acoustical Society of America. [DOI: 10.1121/1.2436630]
\end{abstract}

PACS number(s): 43.30.Re, 43.30.Zk, 43.30.Cq, 43.60.Dh [DRD] Pages: 1895-1908

\section{INTRODUCTION}

It is generally accepted that the ocean environment impacts acoustic communication performance. What is largely unknown is which factors have the strongest impact on performance (e.g., noise, sea state, mixed layer depth, etc.), keeping in mind that they may be signaling method dependent. Also not well known is how to best adapt communication parameters to minimize the impact of these factors. Unlike line-of-sight communications, the shallow-water acoustic ocean channel often gives rise to significant multipath energy caused by reflected and refracted propagation paths between the source and receiver. These time-dispersed multipaths cause replicas of previously transmitted symbols to interfere in the detection of the current symbol and consequently, if strong enough, will result in bit errors.

There are two purposes of this research: (1) to determine, through measurements and modeling, the impact of source/receiver geometry and various environmental factors on shallow-water communications performance and (2) to demonstrate that modeling, with sufficient environmental information, can be used for precise, quantitative performance predictions.

A channel simulator with an embedded high-fidelity acoustic model is used to reproduce both the multipath structure and ultimately the communications performance. Accurate modeling allows the results to be generalized to other sites and environmental conditions, as well as the determination of optimal source/receiver placement. Determining the

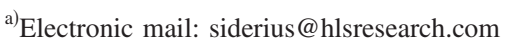

environmental impact on performance is important for predicting when and where underwater communication systems suffer degradation and to what extent. A high-fidelity channel simulator allows for virtual experiments in any desired environment or configuration. For example, suppose a communication link is desired between two underwater vehicles deployed in an area that had previously shown good communications performance for source and receivers moored near the seabed. Can it be assumed that there will be good performance even though the vehicles are moving and operating at different depths and possibly in a different season?

Since many environmental factors that influence performance can either be measured in situ or obtained through archival data, as oceanographic and acoustic models improve, so will acoustic communication system performance prediction and enhancement (e.g., recommending preferred source/receiver operating depths for communications). Cognizance of environmental factors that cause communication system degradation will influence how, when, and/or where a system is deployed.

A study of environmental factors that impact communication performance requires experimentation with careful measurement of channel properties (e.g., ocean sound-speed structure, surface roughness, and currents) while simultaneously transmitting communication signals. Further, these experiments must be conducted over a statistically significant time, and measurements must be designed to isolate the environmental parameters of interest. The Kauai Experiment (KauaiEx) was designed exactly for this purpose and took place off the northwest coast of Kauai, HI in June and July of 2003. ${ }^{1}$ The experiment was designed to measure the environ- 


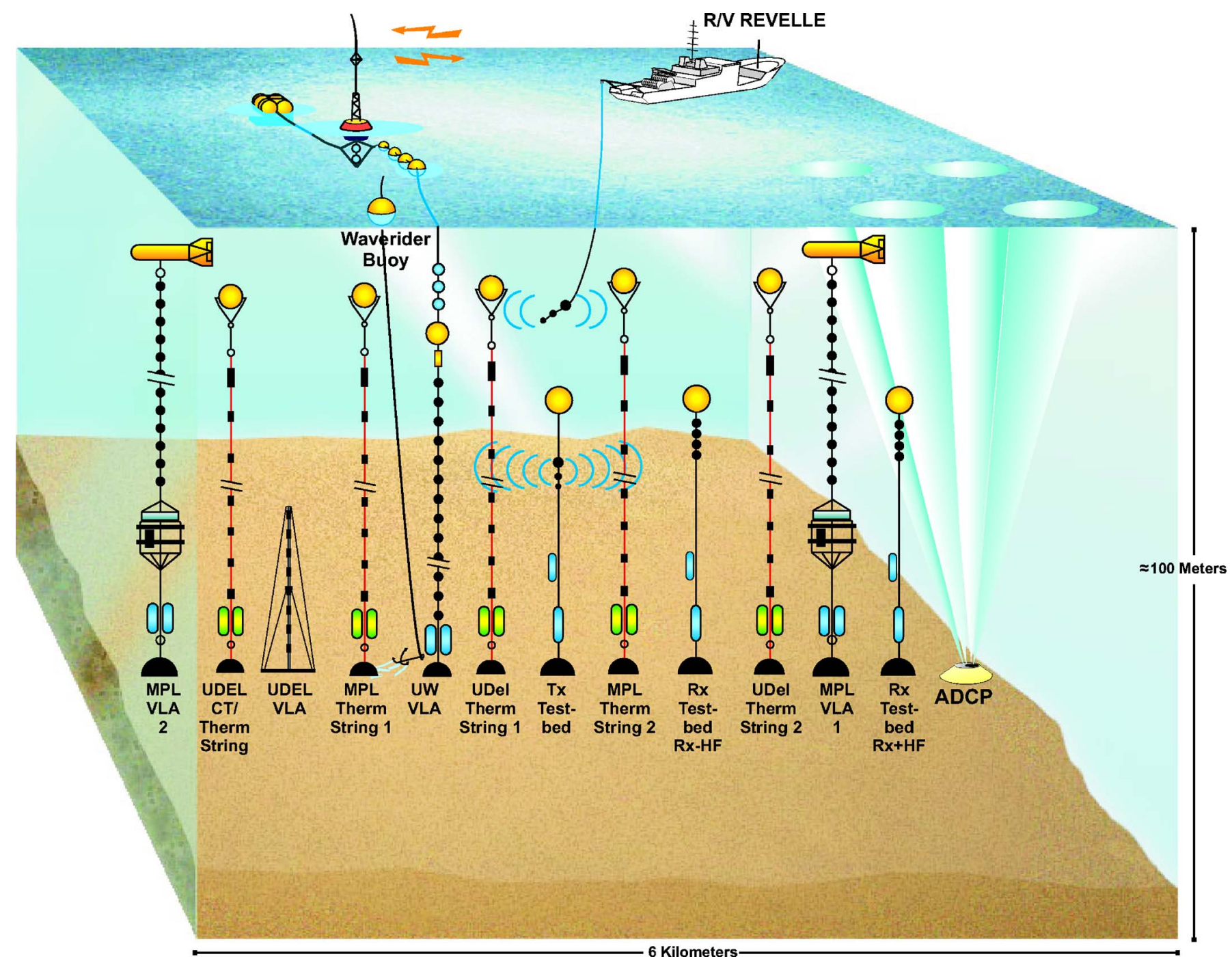

FIG. 1. (Color online) Geometry for the second deployment of KauaiEx. Acoustic source is labeled as Tx Testbed and the data analyzed in this paper was received on array MPL VLA2. The towed source transmissions from the $R / V$ Revelle were interleaved with the moored source for independent analysis.

ment and simultaneously transmit acoustic communication waveforms over several days. This type of experiment has been of interest in recent years as applications for underwater communications have increased. ${ }^{2-4}$

The KauaiEx experiment site presented dynamic oceanographic conditions, typical of shallow-water littorals. To fully capture these conditions, full water-column measurements of both the acoustics and oceanographic properties were conducted simultaneously. Data were collected over many days to obtain significant statistics. Although there were a variety of phases in KauaiEx, this paper focuses on a fixed source and fixed, vertical, receive-array configuration. This provides a much better isolation of the time-varying channel characteristics without having to unravel performance differences caused by changing bathymetry or Doppler effects, as an example. Further, the full-water column vertical array allows different receiver depths to be analyzed simultaneously using a single transmission source. These types of careful, simultaneous, environmental and communication measurements are also required to validate models that simulate the channel characteristics and predict communication performance.
This paper analyzes performance of communication signals using frequency-shift-key modulation $(\mathrm{FSK})^{5}$ that is often implemented in commercial modems because of its robustness and implementation simplicity, especially in the receiver. A general review of various underwater acoustic communications techniques and performance can be found in an overview article by Kilfoyle and Baggeroer. ${ }^{6}$ Because of their implementation in undersea networks, ${ }^{7}$ FSK modems are of practical interest as well. Coherent methods, such as quadrature phase shift keying offer higher spectral efficiencies than noncoherent (i.e., FSK for one) methods. If pointto-point data throughput is an important design consideration, then coherent techniques should be considered for the highly, band-limited underwater acoustic channel. Bandwidth limitations are determined by absorption and are approximately $1 \mathrm{~dB} / \mathrm{km}$ for the $8-13 \mathrm{kHz}$ band considered in this paper. The absorption roughly increases with frequency squared. In addition, the high resonant quality of electroacoustical transduction equipment also limits available bandwidth. However, bandwidth-efficient coherent methods come at the price of processing complexity at the receiver needed to overcome channel variability, and generally require a 
higher signal-to-noise ratio (SNR). In addition to being valuable in its own right, the simple and robust nature of FSK signaling makes its performance a useful yardstick for other methods to compare against.

The balance of this paper is organized in the following manner. Section II describes the Kauai experiment and the data used for analysis. The environmental measurements and the transmitted acoustic communications signals are described. Section III presents the measured performance and that expected assuming both fading and nonfading channel models. This section illustrates the communication system performance impact of source/receiver geometry, watercolumn temperature structure, and wind speed. In Sec. IV a channel simulator with embedded ocean acoustic model is used to replicate and explain measured performance and confirm the channel fading statistics.

\section{THE KAUAI EXPERIMENT}

Details of all experiments that comprise KauaiEx are described by Porter et al. ${ }^{1}$ This paper is based on data collected during the second deployment, 30 June to 3 July 2003. The instruments and their locations are shown in Fig. 1. The towed and moored sources transmitted nonoverlapping acoustic waveforms. Data analyzed here are from the moored Telesonar Testbed only (indicated as Tx Testbed near the middle of the track in Fig. 1) with the source located about $5 \mathrm{~m}$ from the seabed. The Telesonar Testbed is a versatile, wideband, acoustic communication research instrument that has been the centerpiece of many acoustic communication experiments. ${ }^{8}$ The moored Testbed used a subsurface float to maintain the position of the sound projector. Receptions were recorded on multiple arrays but here the data are analyzed from the MPL-VLA2 (Marine Physical Laboratory) receiver array located $3 \mathrm{~km}$ from the source. The vertical line receive array (VLA) was moored and configured with 16 hydrophones spaced $5 \mathrm{~m}$ apart with the first hydrophone about $8.5 \mathrm{~m}$ from the seabed.

As can be seen from Fig. 1, there were extensive environmental measurements including five strings of either thermistors or CTD (conductivity, salinity, depth) sensors to measure water column properties along the acoustic propagation path between transmitter and receiver. In addition, a waverider buoy measured wave heights, and an acoustic Doppler current profiler (ADCP) measured the volumetric water currents. Other geophysical measurements such as grab samples, seismic profiling, and multibeam mapping were also made to help characterize the seabed.

From an acoustic propagation point of view, the bathymetry and seabed along the acoustic propagation path were fairly benign. The acoustic path was over an area believed to be a submerged beach. The grab sample analysis and subsequent visual observation showed it to be mostly a medium grain coral sand with bits of larger coral mixed in. Away from the track, the bathymetry dropped off to several kilometers depth and near the shore decreased to less than $10 \mathrm{~m}$. However, the bathymetry along the propagation path was almost uniform at $100 \mathrm{~m}$.

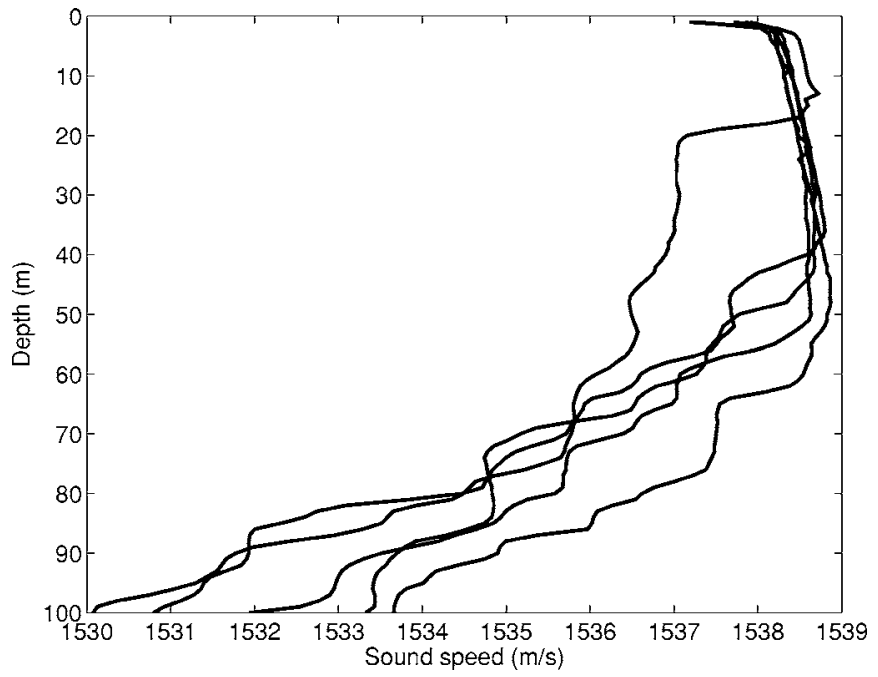

FIG. 2. Measured sound speed profiles taken on 1 July 2003 near the experimental area. Note the change in depth of the mixed layer.

In contrast to the simple bathymetry and seabed, the oceanographic conditions were relatively complex and dynamic over the experiment duration. The water sound-speed, vertical profile depicted a region near the surface with a high degree of mixing due to the often windy conditions; however, the depth of this mixed layer typically varied from approximately 10 to $60 \mathrm{~m}$ but was sometimes deeper. This can be seen from the CTD casts made on 1 July 2003 shown in Fig. 2. Here, the sound speed shows the general trend to decrease with water depth but the depth where the mixed layer ends and the thermocline begins varied with location and time. In these five CTD casts, the mixed layer depth is between 40 and $50 \mathrm{~m}$ for four of the profiles and decreases to about $20 \mathrm{~m}$ for one.

In some locations around the world's oceans, the sound speed near the surface is highly variable due surface warming effects; however, at the KauaiEx site, the wind-driven mixing causes the water near the surface to be more uniform with most of the variability occurring at greater depths. These sound speed profiles give a sense of the structure and variability, but the thermistor strings give a time history for a particular location. In Fig. 3 the data from the thermistor string nearest MPL-VLA2 are shown (it is labeled "UDel CT/Therm. String" in Fig. 1 and is about $500 \mathrm{~m}$ from the VLA). There were 13 thermistors located at depths between 4 and $82 \mathrm{~m}$. There is a clear, regular pattern evident in the thermistor data and shows the time-dependent, thermocline depth variability. It can be seen that in some cases, the thermocline depth is quite shallow and at other times the water column is much more uniform. The impact of these variations on the acoustic communication signals will be discussed in following sections.

\section{EXPERIMENTAL RESULTS AND ANALYSIS}

The FSK signals considered here use 128 frequency components spaced $40 \mathrm{~Hz}$ apart in the $8-13.2 \mathrm{kHz}$ band. The upper and lower 4 tones are reserved for pilot tones to compensate for Doppler. The information is passed using a subset of the 128 frequencies that are modified every $25 \mathrm{~ms}$. 


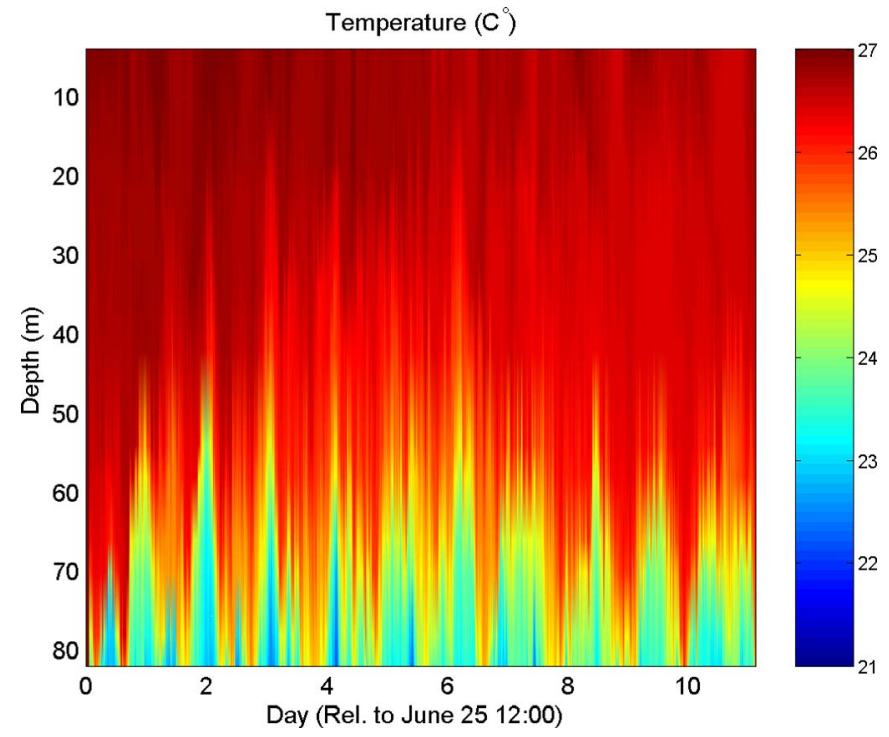

FIG. 3. (Color online) Time history of the ocean temperature during the experiment. This was from the same location on the moored UDel CT/ Therm. string located near MPL-VLA2. Sound speed is mostly a function of the water temperature with a slight dependence on salinity.

The FSK modulation scheme uses 30 instantiations of 1 of 4 coding (i.e., M-ary FSK with $M=4) .^{5}$ This means that 1 of 4 tones activated is used to encode 2 bits of data, i.e., $0-0,0-1$, $1-0$, or 1-1. A practical decoding advantage is gained by requiring the receiver to simply determine which of 4 tones is loudest. This method is less problematic than on/off keying where the decoder decides if a tone is a 1 (on) or 0 (off). This requires thresholding which is very sensitive to channel fading.

Thirty blocks of 4 tones are transmitted simultaneously producing 60 bits in $25 \mathrm{~ms}$, or 2400 bits per second (bps). At the receiver, a spectrogram is taken of the FSK payload (i.e., excluding pilot tones and acquisition components of the transmission packet) using a nonoverlapping boxcar window of $25 \mathrm{~ms}$. The strongest tone in each block of 4 tones is then determined. The ocean, of course, acts like an echo chamber producing multipath spread. To combat multipath spread, the tone duration is increased and the energy over the longer interval is accumulated before conjecturing which tone from the group of four was transmitted. This in turn means a data rate loss. For instance, increasing the tone duration to $50 \mathrm{~ms}$ (by adding two $25 \mathrm{~ms}$ blocks to maintain frequency separation) yields a data rate of $1200 \mathrm{bps}$.

Another component of the modem design is the acquisition process used for initial symbol alignment. There are many ways to do this with pros and cons for each. For these data a set of $m$-sequences proceeding the data payload were match filtered at the receiver to provide symbol time alignment.

Error correction coding at the transmitter is an effective way to reduce errors in fading channels. ${ }^{5,9}$ Although errors can be significantly reduced by coding, this paper considers raw bit errors to reduce the time period over which significant statistics can be developed for adequate analysis. Finally, it should be noted that Doppler effects due to source/
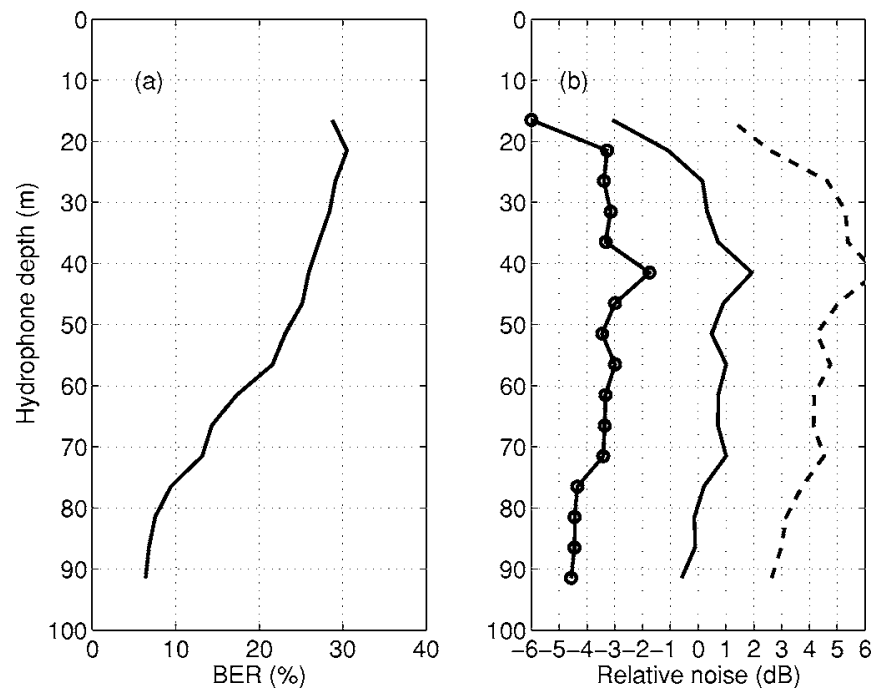

FIG. 4. (a) The depth-dependent percent bit errors averaged over about 1 day of transmissions. (b) The ambient noise as a function of depth averaged over the same time period in three frequency bands: $8-13.2 \mathrm{kHz}$ (solid line), $5-8 \mathrm{kHz}$ (dashed line), and $13.2-16.2 \mathrm{kHz}$ (circles).

receiver motion are typically important for mobile platforms. However, the focus here is on fixed networks so such effects will not be addressed.

\section{A. Measured FSK performance}

One of the most notable observations during KauaiEx was the performance improvement with hydrophone depth. This can be seen in Fig. 4(a) where the bit errors as a function of depth are averaged over about 1 day for $2400 \mathrm{bit} / \mathrm{s}$ transmissions. The deepest hydrophone at approximately $91.5 \mathrm{~m}$ shows an average of about $5 \%$ bit errors while the most shallow at roughly $16.5 \mathrm{~m}$ averages about $30 \%$. One thought might be the lower bit errors are a result of increased SNR due to a decrease in the ambient noise level with depth (transmit level was held constant). However, this is not the case. Shown in Fig. 4(b) is the ambient noise averaged over the same period for the in-band $(8-13.2 \mathrm{kHz})$, below-band $(5-8 \mathrm{kHz})$, and above-band $(13.2-16.2 \mathrm{kHz})$ frequency regions. The figure is on a relative $\mathrm{dB}$ scale and shows the roughly $6 \mathrm{~dB}$ decrease in noise as frequency is doubled. Also, there is little evidence that the noise conditions are improved at lower depths; actually, the shallowest hydrophone shows the lowest noise level. Nevertheless, the data show that, in general, the noise field is relatively homogeneous, vertically. It turns out that the improvement in performance with depth is partly due to higher signal level rather than lower ambient noise levels (another factor is the multipath which is analyzed in detail in later sections). The higher signal levels are caused by the thermocline trapping acoustic communication signals near the sea floor; this will be discussed further in Secs. III E and III F.

There is also an interesting difference in the temporal variability of performance at different receiver depths observed over a 24 hour period which captures diurnal oceanographic and wind cycles. Figure 5(a) shows time history of the average bit errors for the four shallowest hydrophones at 16.5, 21.5, 26.5, and $31.5 \mathrm{~m}$ for 2400 bit/s rate. In Fig. 5(b) 

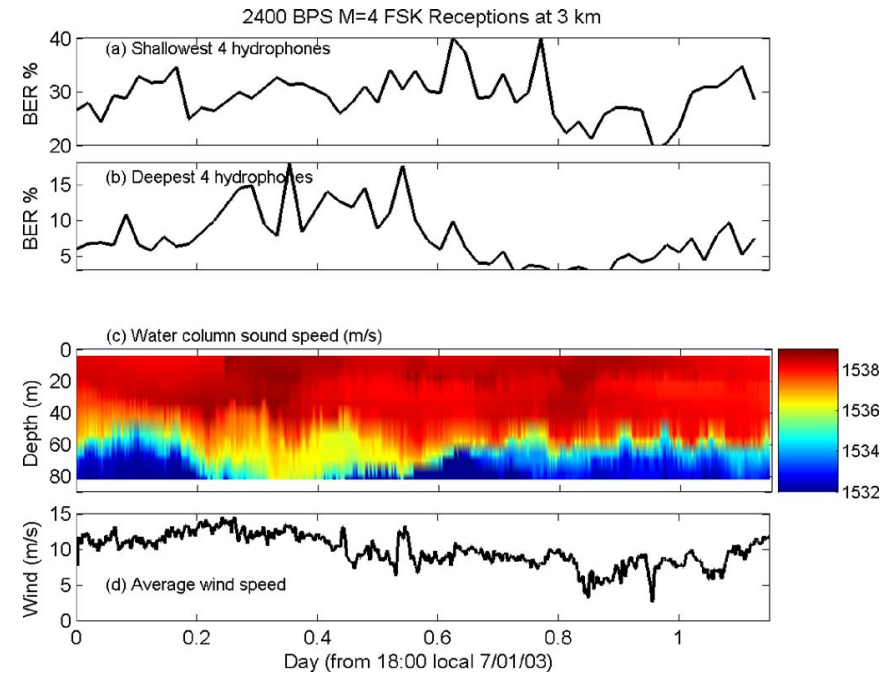

FIG. 5. (Color online) (a) The averaged percent bit errors for the shallowest four hydrophones for $2400 \mathrm{bps}$ transmissions over about 1 day. In (b), average bit errors are shown for the deepest four hydrophones [note the different $y$ axis from (a)]. In (c), the water column sound speed is shown and in (d) the average wind speed during the same period. The $x$ axis is slightly more than 1 day referenced to 18:00 on 1 July 2003 (local time).

the average bit errors for the four deepest hydrophones at $91.5,86.5,81.5$, and $76.5 \mathrm{~m}$ are shown [note the change in the $y$ axis compared to (a)]. The period of the day between about 0.2 and 0.6 shows a marked increase in bit errors for the deepest hydrophones followed by a decrease. Figure 5(c) shows the water column sound speed and Fig. 5(d) shows the wind speed during the same time period. The water sound speed measurement was made by a thermistor string located about $500 \mathrm{~m}$ from the receivers.

A few observations can be made from Fig. 5. First, the period corresponding to the bit error increase for the deepest hydrophones corresponds to the period where the water column is more mixed and the thermocline presence is reduced. The link between bit errors and thermocline position is weaker for the shallower hydrophones. Second, there are more errors overall for the shallow hydrophone $(\sim 30 \%)$ compared to the deep hydrophone $(\sim 5 \%)$. The resultant temperature-dependent channel conditions account for this phenomenon. The percent bit errors for the deepest hydrophones increase when the water column becomes more mixed due to upper, warmer water moving deeper. This creates a sound-speed profile without a thermocline and therefore without the mechanism to trap acoustic signals. This is more evident when looking at modeling results in Sec. IV and at the impulse response measurements described in Sec. III F.

Wind speed is fairly constant during the $0.2-0.6$ period; however, in the $0.8-1.0$ time period the wind speed decreases below $5 \mathrm{~m} / \mathrm{s}$ with a corresponding decrease in bit errors for both the shallow and deep hydrophones. The winds obviously affect both the ambient noise and the surface scatter loss. It is reasonable to wonder whether surface scatter will attenuate the steeper angle paths and thereby reduce multipath leading to a corresponding reduction in inter symbol interference (ISI). One might then also wonder if better modem performance can be expected with surface scatter.
However, surface scatter is a complicated subject. Surface losses can be due to (1) a static surface that scatters energy in different directions, ultimately leading to higher losses through bottom absorption and (2) a perfectly flat but dynamic surface that distorts the FSK tones and produces a weaker coherent signal. The effects of the latter depend also on the tone duration since a short tone effectively freezes the surface. In fact, both these mechanisms are in play; however, in acoustic modeling these distinct mechanisms are often treated vaguely as one. Although not conclusive, the measurements here do not appear to support an argument that increased roughness improves modem performance. Actually, the performance improves during periods with calmer seas and lower wind speed. However, in these data the variations in the sound speed profile seem to dominate.

\section{B. Predicted FSK performance}

The theory of FSK performance for a nonfading channel in the presence of additive noise has been well developed. ${ }^{5}$ As background to the discussions here, a review of those results is presented with the notation closely following the derivation presented in Proakis. ${ }^{5}$ This analysis generally holds for FSK transmissions but concentrates specifically on the $M=4$ FSK that was used during KauaiEx.

\section{Nonfading channel model}

The signal sinusoidal tones are "on" for the bit duration $T_{b}$ and are expressed as

$$
s(t)=\sqrt{\frac{2 \mathcal{E}_{b}}{T_{b}}} \cos (2 \pi f t+2 \pi(m-1) \Delta f t), \quad m=1,2,3,4,
$$

where $f$ is the frequency for $m=1$ and $f+(m-1) \Delta f$ for the neighboring tones. The amplitude is expressed in terms of the energy per bit $\mathcal{E}_{b}$. In a nonfading channel, the received signal is

$$
\begin{aligned}
& r(t)= \sqrt{\frac{2 \mathcal{E}_{b}}{T_{b}}} \cos (2 \pi f t+2 \pi(m-1) \Delta f t+\phi)+n(t), \\
& m=1,2,3,4,
\end{aligned}
$$

where $\phi$ is the phase shift due to the transmission delay and $n(t)$ is additive white Gaussian noise. To obtain the amplitude of the received signal regardless of the phase, $r(t)$ is correlated with the quadrature carriers, $\sqrt{1 / T_{b}} \cos (2 \pi f t$ $+2 \pi(m-1) \Delta f t)$ and $\sqrt{1 / T_{b}} \sin (2 \pi f t+2 \pi(m-1) \Delta f t)$. The detector selects the largest tone by computing the envelope of the correlations, or

$$
r_{m}=\sqrt{r_{m c}^{2}+r_{m s}^{2}},
$$

where $r_{m c}$ and $r_{m s}$ are the correlation outputs from the cosine and sine components for the $m$ th tone.

For the nonfading channel the on tone components will be denoted as $m=1$ and are simply

$$
r_{1 c}=\sqrt{\mathcal{E}_{b}} \cos \phi_{1}+n_{1 c}
$$


and

$$
r_{1 s}=\sqrt{\mathcal{E}_{b}} \sin \phi_{1}+n_{1 s} .
$$

The "off" tones $(m=2,3,4)$ have: $r_{m c}=n_{m c}$ and $r_{m s}=n_{m s}$ with the noise components $n$ being mutually, statistically independent, zero-mean Gaussian variables with equal variance $\sigma^{2}$ $=N_{0} / 2$. For notational convenience, the random variable $R_{m}$ is defined as

$$
R_{m}=\frac{\sqrt{r_{m c}^{2}+r_{m s}^{2}}}{\sigma}
$$

Next, consider the probability distributions of the amplitudes for the on and off tones. The on tone amplitude probability distribution is Ricean and written as

$$
p_{R_{1}}\left(R_{1}\right)=R_{1} \exp \left[-\frac{1}{2}\left(R_{1}^{2}+\frac{4 \mathcal{E}_{b}}{N_{0}}\right)\right] I_{0}\left(\sqrt{\frac{4 \mathcal{E}_{b}}{N_{0}}} R_{1}\right),
$$

where $I_{0}$ is the zeroth-order modified Bessel function of the first kind. For the $m=2,3,4$ terms the probability distributions are Rayleigh,

$$
p_{R_{m}}\left(R_{m}\right)=R_{m} \exp \left(-\frac{1}{2} R_{m}^{2}\right) .
$$

A correct decision will be made if $R_{1}>R_{m}$ or

$$
P_{c}=\int_{0}^{\infty}\left[P\left(R_{1}>R_{m} \mid R_{1}=x\right)\right]^{3} p_{R_{1}}(x) d x,
$$

where

$$
P\left(R_{1}>R_{m} \mid R_{1}=x\right)=\int_{0}^{x} p_{R_{m}}\left(r_{m}\right) d r_{m}=1-e^{-x^{2} / 2},
$$

and the power of 3 arises from the fact that for $m=2,3,4$ the random variables are statistically independent and identically distributed so the joint probability factors into a product,

$$
P_{c}=\int_{0}^{\infty}\left(1-e^{-x^{2} / 2}\right)^{3} p_{R_{1}}(x) d x .
$$

The general solution to Eq. (11) is given in Proakis ${ }^{5}$ and for $M=4$ is

$$
P_{c}=\sum_{n=0}^{3}(-1)^{n} \frac{3 !}{n !(3-n) !(n+1)} \exp \left[\frac{-2 n \mathcal{E}_{b}}{(n+1) N_{0}}\right] .
$$

Finally, the probability of a bit error is

$$
P_{\mathrm{BE}}=\frac{2}{3}\left(1-P_{c}\right) \text {. }
$$

The factor of $\frac{2}{3}$ provides the additional reduction in errors that accounts for only using one out of four tones to convey 2 bits.

\section{Fading channel model}

A fading channel model can cause an average decrease in received signal-to-noise ratio (over that of the nonfading channel) which leads to higher bit errors. In addition, the fading causes the amplitudes of the on and off bits to vary in such a way that additional bit errors are produced. In the presence of multipath, ISI causes a bleed which may make the off bits appear to be on. In other words, the multipath causes the signal to appear in adjacent time bins which is effectively another noise mechanism. Further, both the noise and signal amplitudes vary due to the multipath interference. Following Proakis, ${ }^{5}$ for the slowly fading channel, the received signal for the on bits is attenuated by a factor of $\alpha$, that is, $r(t)=\alpha s(t)+n$. The energy per bit to noise is effectively $\gamma_{b}=\alpha^{2} \mathcal{E}_{b} / N_{0}$. The probability of an error can be predicted by modifying Eq. (13) to incorporate the new SNR. But, for the fading channel, $\alpha$ is random so the previous estimate for the probability of an error needs to be averaged over the probability density function of $\gamma_{b}, p_{\gamma_{b}}\left(\gamma_{b}\right)$. Thus, to estimate the number of bit errors for the fading channel, $P_{\mathrm{BEF}}$, the error probability for the nonfading channel [given by Eq. (13)] is averaged over $p_{\gamma_{b}}\left(\gamma_{b}\right)$,

$$
P_{\mathrm{BEF}}=\int_{0}^{\infty} P_{\mathrm{BE}}\left(\gamma_{b}\right) p_{\gamma_{b}}\left(\gamma_{b}\right) d \gamma_{b} \text {. }
$$

For Rayleigh fading, $\alpha$ is Rayleigh distributed. This means $\alpha^{2}$ has a chi-square distribution and so too does $\gamma_{b}$. Thus,

$$
p_{\gamma_{b}}\left(\gamma_{b}\right)=\frac{1}{\bar{\gamma}_{b}} e^{\left(-\gamma_{b} / \bar{\gamma}_{b}\right)},
$$

where $\bar{\gamma}_{b}$ is the average energy per bit to noise ratio,

$$
\bar{\gamma}_{b}=\frac{\mathcal{E}_{b}}{N_{0}} E\left(\alpha^{2}\right),
$$

with $E\left(\alpha^{2}\right)$ being the average of $\alpha^{2}$.

This discussion clearly assumes that the multipath structure is sufficient to generate Rayleigh fading. Another fading model uses the Nakagami- $m$ distribution. ${ }^{5}$ This is useful since it allows for a family of bit error probabilities that have fading better than and worse than Rayleigh. Nakagami- $m$ fading has a fading figure parameter $m$ that can be less than 1 for fading situations worse than Rayleigh, equal to one for exactly Rayleigh, and greater than 1 when fading is more favorable than Rayleigh. ${ }^{5}$ The data analysis will show that the Rayleigh fading model is good much of the time, but not universally.

\section{Measured signal-to-noise ratio}

The probability of bit errors, for both fading and nonfading channels is, in part, dependent on the actual or effective SNR (or energy-per-bit to noise ratio). In underwater acoustics, SNR has historically been calculated using the sonar equation, 10

$$
S N R=S L-T L-N,
$$

where $S L, T L$, and $N$ are the source level, transmission loss, and noise levels measured in decibels. This approach works well for sonar applications where signals are often integrated for periods much longer than the duration of multipath. However, for computing SNR levels and consequently predicting communication system performance, the sonar equa- 


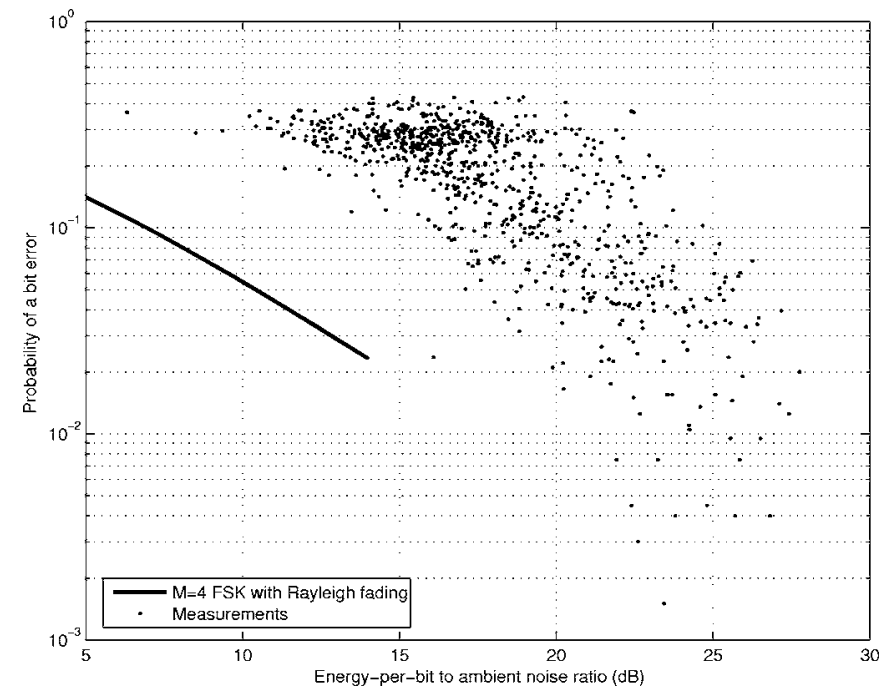

FIG. 6. Measured percent bit errors as a function of the energy-per-bit to ambient noise in decibels (black dots). Also shown is the expected bit error probability for Rayleigh fading with $M=4$ FSK (solid line).

tion approach can be very misleading. To illustrate, the KauaiEx data SNR was calculated this way using the following procedure. The background noise $N$ was measured just below and above the transmission band $(8-13.2 \mathrm{kHz})$. To obtain an in-band noise estimate, these bracketing, but out-of-band levels were interpolated on a log scale. The interpolation on a log scale is used since ambient noise in this frequency band is approximately linear on a log scale $^{10}$ [refer to Fig. 4(b) showing measured ambient noise below, in, and above the transmission band]. The signalplus-noise $(S+N)$ was measured in the band and SNR calculated as

$$
10 \log \left\{\frac{(S+N)-N}{N}\right\} \text {. }
$$

The apparent signal and noise level is obviously reduced when processed through a passband filter. Thus, the signal and noise were scaled to compensate for different bandwidths, and further adjusted by $3 \mathrm{~dB}$ to convert from energy per symbol to energy per bit. The measured SNR (i.e., energy-per-bit to background ambient noise ratio) is plotted against the probability of a bit error in Fig. 6. The measured SNR calculated in this way shows a wide range of bit errors for a given SNR. For instance, at $20 \mathrm{~dB}$ there is a spread in error probability from $<2 \%$ to around $40 \%$. Furthermore, the predicted bit errors using a Rayleigh fading channel model [Eq. (14)] is shown as a solid line in Fig. 6. This would imply a severely fading channel much worse than even a Rayleigh model described in Sec. III B. The measured SNR in Fig. 6 is the energy-per-bit to background ambient noise ratio. This is different from the effective noise that includes multipath, which has the biggest impact on bit errors as will be described in the next section.

\section{Measured energy-per-bit to noise ratio with multipath included}

Due to significant multipath that exists in many shallow water environments, the concept of "signal plus multipath" has been introduced. "The idea being that the "true noise" is a combination of ambient noise plus multipath and "true signal" also contains many arrivals. If there is more than one arrival, i.e., direct and surface bounce paths, they will interfere producing a tone stronger or weaker than either of the individual arrivals (again, remembering that it is assumed that Doppler is not significant in these discussions). In addition, subsequent arrival(s) may "bleed" (ISI) into the next symbol's time slot which may cause an error. The duration and stability of the multipath controls the fading of the shallow water channel.

Theoretical FSK performance for both fading and nonfading channels was shown to be a function of SNR or, more specifically, the energy-per-bit $\left(\mathcal{E}_{b}\right)$ to noise $\left(N_{0}\right)$ ratio. The SNR calculation presented for the KauaiEx data in Sec. III C does not show typical SNR-dependent performance, due in part to multipath interference effects. In fact, the background ambient noise level has little to do with the actual performance in these data. An effective $\mathcal{E}_{b}$ and $N_{0}$ can be measured that also contains the multipath. The energy-per-bit-withmultipath $\left(\mathcal{E}_{b M}\right)$ to noise-with-multipath ratio $\left(N_{0 M}\right)$ is the effective $\mathcal{E}_{b} / N_{0}$ needed to compare with the theoretical predictions and to determine the channel fading statistics. To obtain $\mathcal{E}_{b M} / N_{0 M}$ the same procedure is used as for decoding the data as described in the introduction to Sec. III. An $m$-sequence matched filter is applied to the received signal for aligning symbol timing. A spectrogram with a $T_{b}$-length boxcar window is then computed. This will produce the ontone amplitudes and the amplitude of bins that should be off. The average of all the on bins and all the off bins is a direct measurement of $\mathcal{E}_{b M}$ and $N_{O M}$ and this is used to compare with predicted fading models.

The measured probability of bit error is shown as a function of measured $\mathcal{E}_{b M} / N_{0 M}$ in Fig. 7. Also shown are the theoretical performance for $M=4 \mathrm{FSK}$ in a nonfading [Eq. (13)] and Rayleigh fading channel [Eq. (14)]. As can be seen, the data roughly fall on the curve predicted for a Rayleigh fading channel; however, the fading is slightly worse at low values of $\mathcal{E}_{b M} / N_{0 M}$ and slightly better at high $\mathcal{E}_{b M} / N_{0 M}$. Further, the figure has a color coding which shows the dependency on hydrophone depth and indicates that the $\mathcal{E}_{b M} / N_{0 M}$ is lower for the shallow hydrophones and the bit errors are higher.

Interestingly, in Fig. 7, the best performance at the highest $\mathcal{E}_{b M} / N_{0 M}$ ratios has a fading characteristic that is much better than the Rayleigh model yet not quite as good as a nonfading channel. These, isolated data, were fit to a Nakagami fading model with a 1.5 fading factor. This indicates times when the channel had a dominant arrival and results were closer to the nonfading channel. Results in Fig. 7 indicate that better performance is achieved through larger $\mathcal{E}_{b M} / N_{0 M}$ but this does not imply higher source level. The performance degradation occurs because of multipath and not the ambient noise level so only a more favorable geom- 


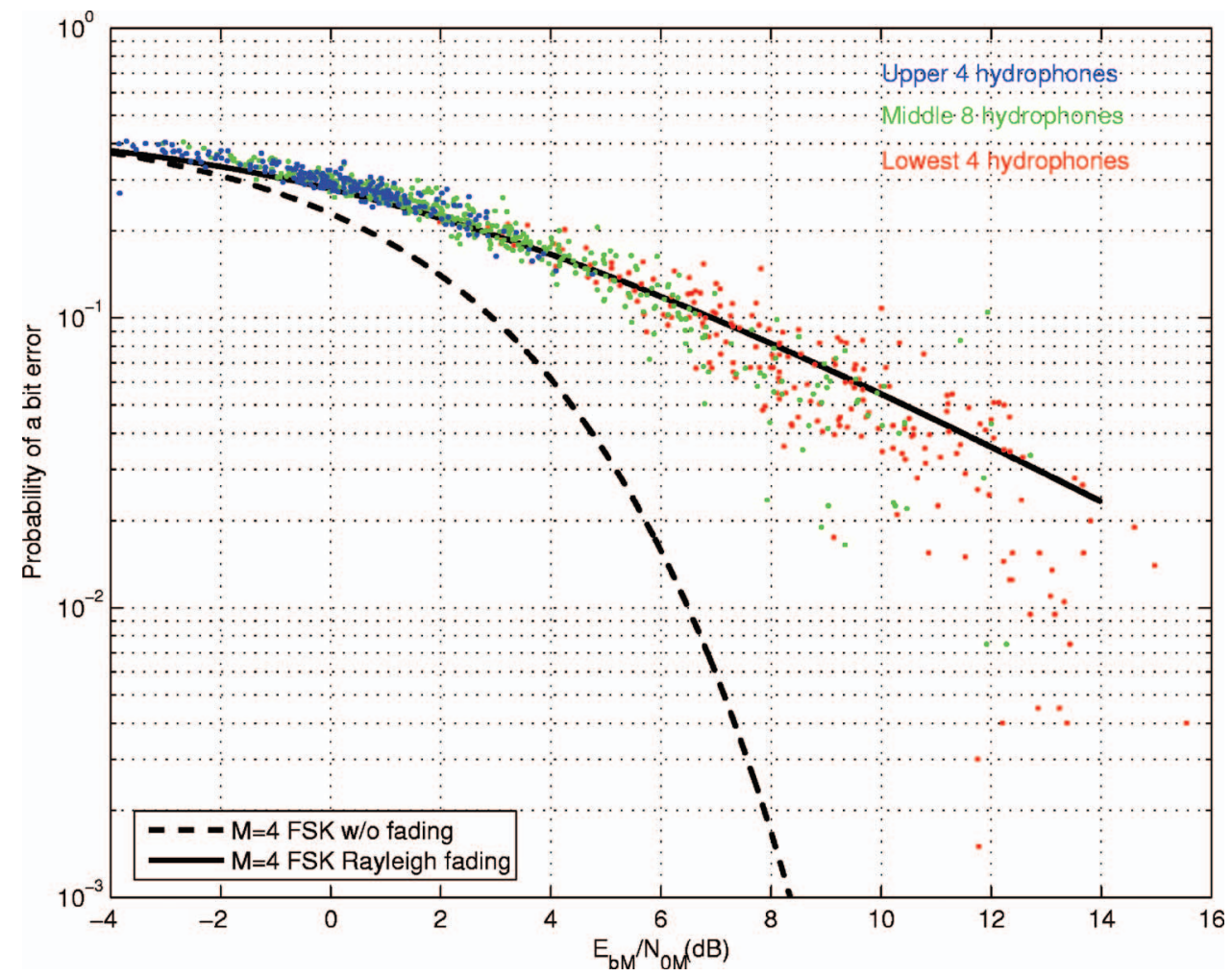

FIG. 7. Probability of bit error for transmissions during 1 day of KauaiEx vs $\mathcal{E}_{b M} / N_{0 M}$. The solid line is the theoretical performance for a Rayleigh fading channel and the dashed line is for a nonfading channel. The colored dots indicate receiver depth within the water column. The blue dots are the shallowest hydrophones, the green dots are in the middle of the water column, and the red dots are the deepest hydrophones.

etry (or different environmental conditions) can improve performance. This is indicated by observing the color coded points in Fig. 7 showing different $\mathcal{E}_{b M} / N_{0 M}$ at different receive depths with the same source level. This point will be demonstrated further in Sec. IV.

\section{E. Measured and predicted channel fading}

The envelope of the measured amplitude distributions for shallow and deep hydrophones taken over the 1 day experiment is shown in Fig. 8 as solid black and gray lines. The distributions are also fit to the best Rayleigh curves and those are shown as dashed lines. Figure 8 shows the shallow hy-

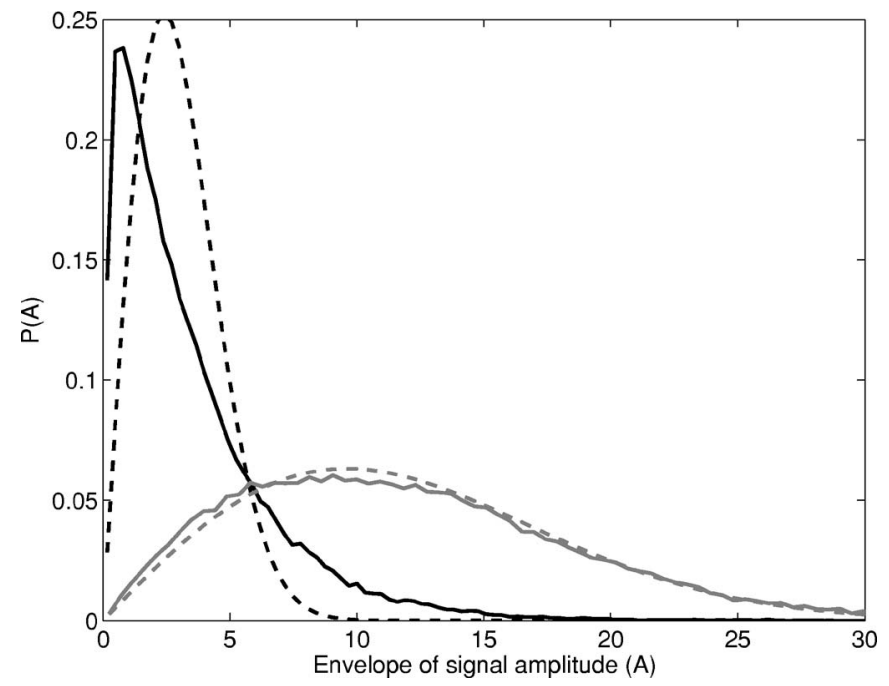

FIG. 8. The envelopes of the amplitude distributions for shallow (16.5 m, black solid curve) and deep ( $86.5 \mathrm{~m}$, gray solid curve) hydrophones averaged over the 1 day experiment. Also shown are the curves that best fit using a Rayleigh distribution (dashed lines). drophone has a distribution that is worse than Rayleigh. That is, the amplitudes are smaller being clustered more tightly near zero. For hydrophones at these depths a more general fading model that uses, for example, the Nakagami- $m$ distribution (with an $m$ parameter less than 1 for more severe fading than Rayleigh) may provide a better prediction. However, the intermediate and deep hydrophone are fit very well to a Rayleigh curve. These distributions are consistent with the bit error probabilities, that showed slightly worse than Rayleigh fading on the shallow hydrophones and nearly Rayleigh fading on the deep hydrophones.

Although the distribution for the deep hydrophones is approximately Rayleigh when averaged over the 1 day experiment, there is less agreement when considering shorter time scales. Figure 9 depicts two curves corresponding to the envelope of the amplitude distribution when signaling on one of the deep hydrophones. Each curve represents three $0.8 \mathrm{~s}$ transmissions occurring at two different times: (1) the gray dashed line corresponds to a time frame when the water column is well mixed, nearly no thermocline, and incidentally also corresponds to higher error rates and (2) the black dashed line corresponds to a time frame when the thermocline is well established, and also corresponds to lower error rates. The solid lines are the best-fit curves and demonstrate that during the mixed period with high error rates, the distribution is closely fit to Rayleigh; on the other hand, a Ricean (Sec. III B 1) curve fits best to the period with a well-defined thermocline and corresponding lower error A rates.

To summarize, the Rayleigh fading model is useful to explain much of the data; however, it is not universally applicable. In particular, it is not applicable when there is a dominant arrival as was the case for deep receivers with a 


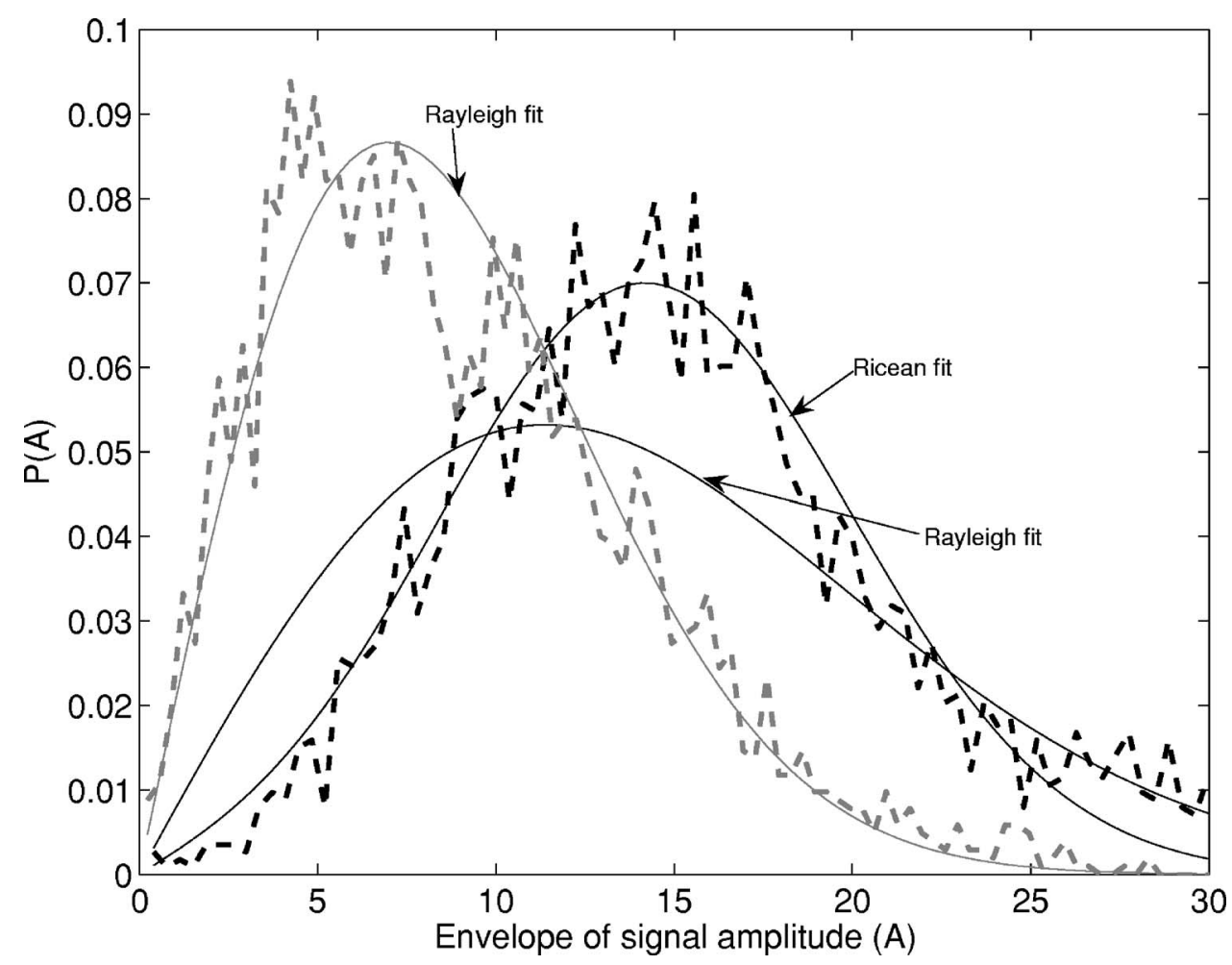

FIG. 9. Envelopes of the amplitude distributions on a short time scale (data are taken from 3, $0.8 \mathrm{~s}$ transmissions) for a deep hydrophone at $86.5 \mathrm{~m}$. During the period with the water column mixed, the errors increased and the amplitude distribution is represented by the gray dashed curve, with the solid gray being the best fit to a Rayleigh distribution (higher BER). For the period with a strong thermocline, there were much fewer errors and the amplitude distribution for that time is shown as a black dashed line with the best fit Rayleigh and Ricean curves as solid black lines (lower BER).

well-established thermocline. Further, it is important to consider the time scale over which a statistic is desired. Modem performance on a very long time scale is more likely to average through periods where there is a dominant multipath. The statistics over the long time will then be characterized by the Rayleigh fading model. In contrast, predicting performance over a short period may require taking into account a situation with a single dominant multipath. The short-time scenario might be an AUV deployed for a few hours, while the long-time scenario might be for a fixed networked deployed for many months.

\section{F. Impulse response measurements}

The channel impulse response is one of the most important measurements for understanding propagation physics. Snapshot impulse response measurements reveal instantaneous multipath structure, duration, and strength; taken over time, these measurements often show the impulse response time-variability due to changing environmental conditions. These time-stacked impulse responses can be used to understand the amplitude distributions and how they impact performance.

A matched filter was applied to $50 \mathrm{~ms}, 8-16 \mathrm{kHz}$, linear frequency modulated (LFM) probe signals that were transmitted during KauaiEx to provide an equivalent, bandlimited impulse response. Matched filtering was implemented for each receiver on the vertical array. In Fig. 10, an example is shown of measured impulse responses for periods corresponding to times with low (a) and high (b) bit errors. Figure 10 clearly shows multipath arrivals with duration of 50-100 ms, which is greater than the FSK symbol length of $25 \mathrm{~ms}$. The figures have a $30 \mathrm{~dB}$ dynamic range scaled by the largest values. For the data in Fig. 10(a), there is a region around $0.025 \mathrm{~s}$ on the deeper hydrophones that shows a much larger amplitude arrival relative to the others. In Fig. 10 (b) note that there is no one dominant arrival.

\section{PERFORMANCE PREDICTION USING A CHANNEL SIMULATOR}

In recent years, advances have been made in using physics based, propagation modeling to simulate the channel impulse response and communications performance. ${ }^{11,12}$ However, there have been very few experiments with simultaneous acoustic and environmental measurements to the extent taken during KauaiEx. These simultaneous measurements are needed for model validation and model/data comparisons. The simulation tool used for comparing measured data with modeled results is based on the Gaussianbeam tracing code BELLHOP ${ }^{13}$ with an added feature to allow for moving platforms (i.e., Doppler effects). ${ }^{14}$ This added feature, which produces different Doppler on each propagation path, is not exploited here since the source and receiver are stationary. ${ }^{14}$ This simulator can be used with any communications signal in environments that vary volumetrically. That is, variable bathymetry and seabed properties, and depth- and range-dependent sound speed can be included for both coherent and noncoherent simulated transmissions.

Only the static case (i.e., simulations with source and receivers in fixed positions) will be described here. In this case, the BELLHOP model produces a set of arrivals each with the appropriate time delay and a complex amplitude. To describe the process of obtaining simulated communication transmissions, begin by noting that the complex pressure field, $P(\omega)$, can be represented as a sum of $K$ arrival amplitudes $A_{k}(\omega)$ and delays $\tau_{k}(\omega)$ according to 

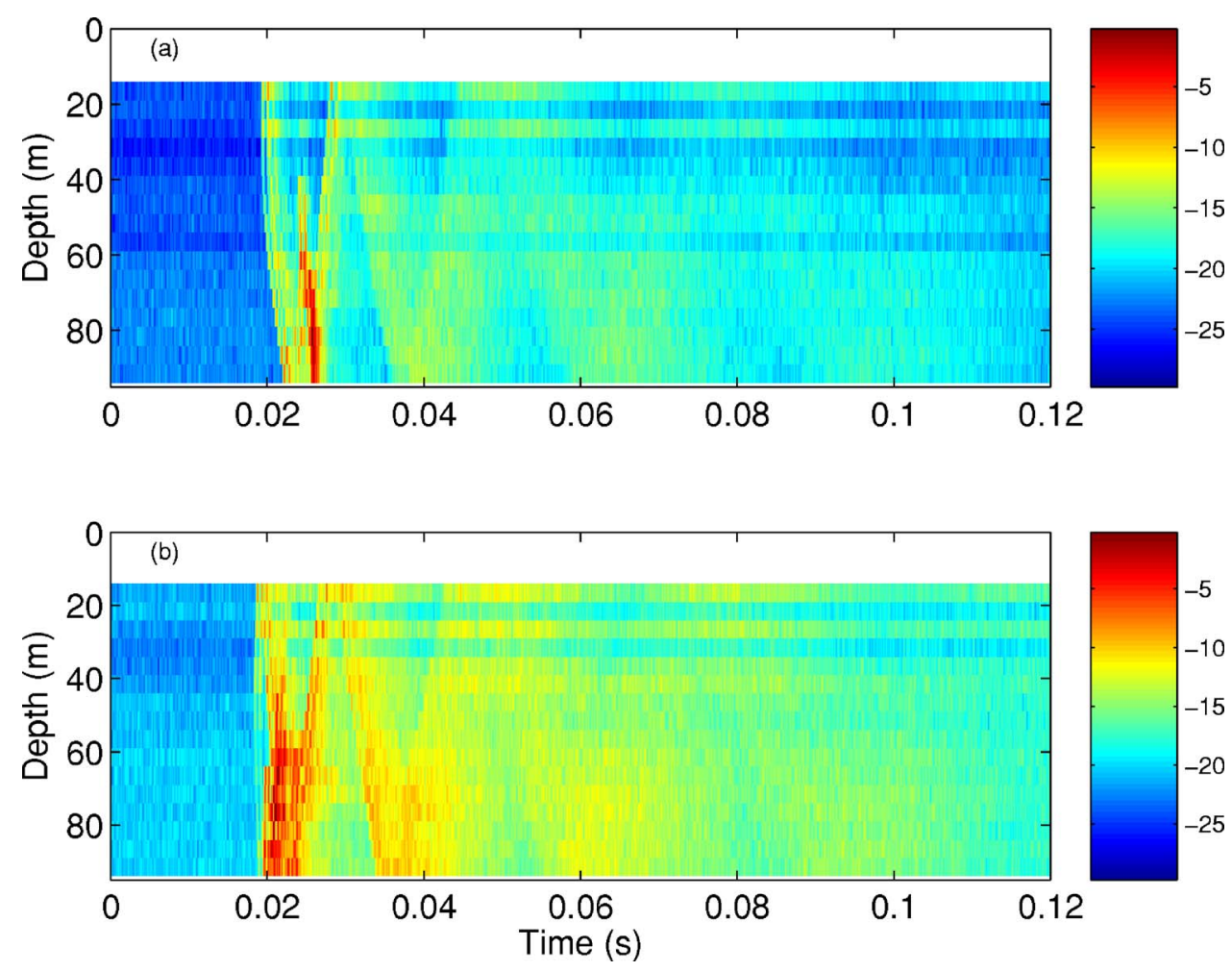

FIG. 10. (Color online) Measured impulse responses (magnitude) from matched-filtered LFM transmissions at times with low and high bit errors. Each panel is on a relative scale of $30 \mathrm{~dB}$ with the highest overall peak set to $0 \mathrm{~dB}$. Panel (a) corresponds to a period with bit errors less than 5\% (day 0.15) and panel (b) to errors close to $20 \%$ (day 0.35). The notable feature in (a) is the focusing of arrivals at the deepest hydrophones that produces a peak, in time, much larger than the other paths.

$$
P(\omega)=S(\omega) \sum_{k=1}^{K} A_{k} e^{-i \omega \tau_{k}}
$$

The convolution theorem states that a product of two spectra is a convolution in the time domain. This leads to the corresponding time-domain representation for the received wave form, $p(t)$, which is often written as

$$
p(t)=\sum_{k=1}^{K} A_{k} s\left(t-\tau_{k}\right)
$$

where $s(t)$ is the source wave form. This representation is very intuitive, showing the sound that is heard as a sum of echoes with various amplitudes and delays. However, the amplitudes are complex to account for the interactions with the seabed and the additional time delays introduced. A more careful application of the convolution theorem considers the complex amplitudes and the conjugate symmetry of $P(\omega)$ which is necessary to guarantee a real received wave form. The proper result is then

$$
p(t)=\sum_{k=1}^{K} \operatorname{Re}\left\{A_{k}\right\} s\left(t-\tau_{k}\right)-\operatorname{Im}\left\{A_{k}\right\} s^{+}\left(t-\tau_{k}\right),
$$

where $s^{+}=\mathcal{H}(s)$ is the Hilbert transform of $s(t)$. The Hilbert transform is a $90^{\circ}$ phase shift of $s(t)$ and accounts for the imaginary part of $A_{k}$. Equation (21) states that any arbitrary phase change can be understood as a weighted sum of the original wave form and its $90^{\circ}$ phase-shifted version. The weighting controls the effective phase shift which occurs at bottom reflections and can yield arbitrary phase shifts. Additionally, paths that refract within the water column can be distorted in a similar way as the waves pass through caustics. It should be noted that for these simulations the seabed is treated as an infinite halfspace which is reasonable since in the communications frequency band there is minimal penetration into the seabed. The half-space representation allows for a single ray trace to be used when constructing the broadband time series. This allows for rapid calculation of these highfrequency, broad band transmissions.

Simulations for KauaiEx. During the second major deployment during KauaiEx, the source was located at $95 \mathrm{~m}$ depth and the 16-element receiver array was $3 \mathrm{~km}$ away at depths of $16.5-91.5 \mathrm{~m}$ in $5 \mathrm{~m}$ increments. For the simulations, the seabed properties used were compressional sound speed of $1600 \mathrm{~m} / \mathrm{s}$, attenuation of $0.5 \mathrm{~dB} / \lambda$, and density of $1.8 \mathrm{~g} / \mathrm{cm}^{3}$. The ray traces between the source and the deepest hydrophone are shown in Fig. 11 for the two time periods previously discussed, that is, low and high bit errors. The bit error for just the deepest hydrophone at $91.5 \mathrm{~m}$ is shown in the top panel of Fig. 11 and the middle panel depicts the water-column sound speed structure during the same period. During the first period, the thermocline existed well above the hydrophones and during the second, the thermocline was absent (or nearly).

Impulse response simulations. Fifty millisecond LFM transmissions from 8 to $16 \mathrm{kHz}$ were simulated and matched filtered in the same way the measured impulse responses were processed. These impulse response plots are shown in Fig. 12 and can be compared with the measured impulse responses shown in Fig. 10. In these cases, the water column sound-speed profile measured near the VLA was used for times closest to the measured impulse responses shown in Fig. 10. Note the strong focused region for the deeper hydrophones and the similarity to the measurements. 


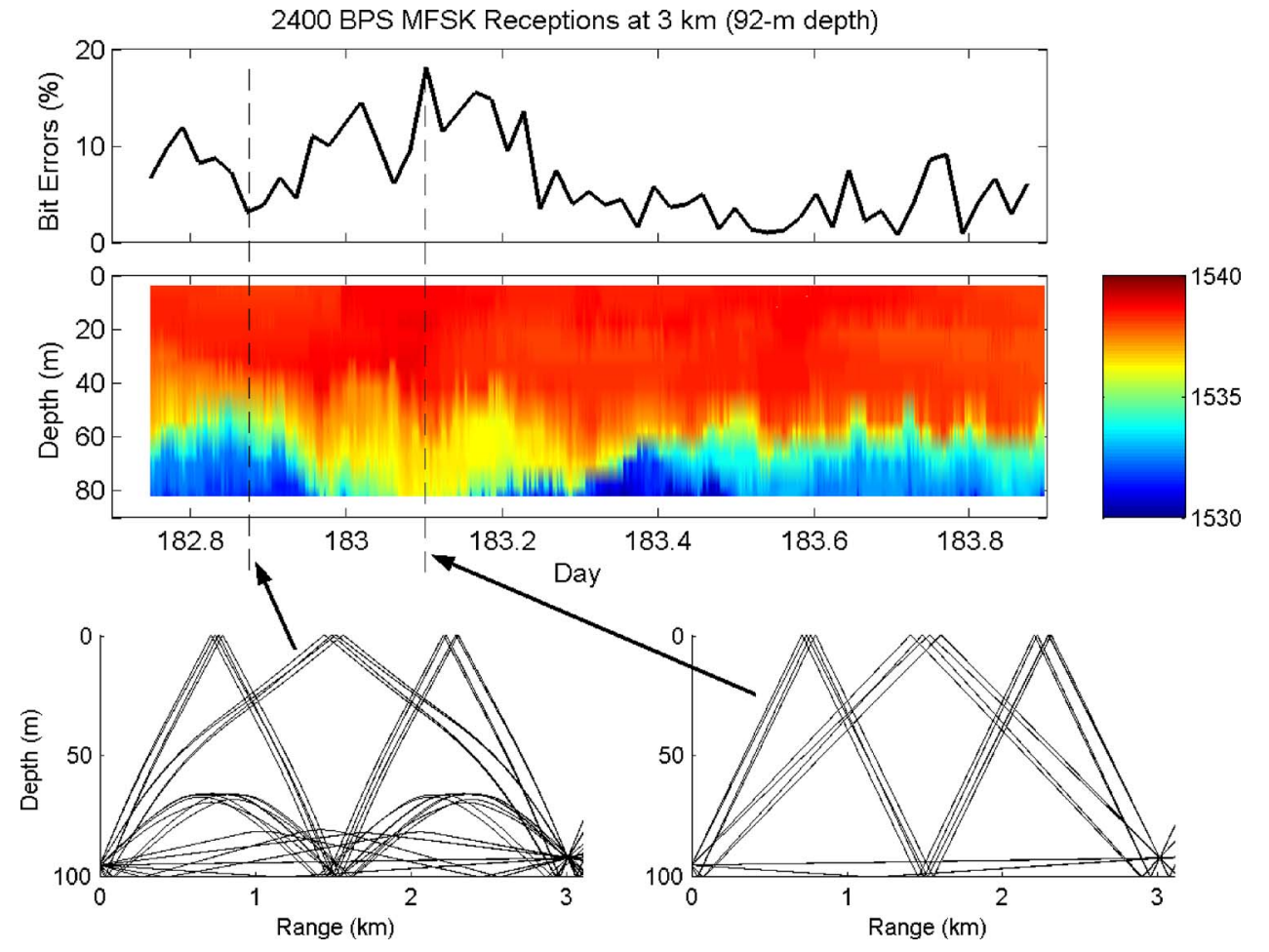

FIG. 11. (Color online) Top panel shows the percent bit errors for 2400 bps transmissions over about 1 day using the deepest hydrophone at $91.5 \mathrm{~m}$. The middle panel shows the corresponding water column sound speed profile. The lowest left panel shows the ray trace that corresponds to the time with a thermocline (lower error) and the lower right panel for mixed water column (higher errors).

Simulations of FSK fading and performance. Communication signals as input to simulators can provide insight into observed performance behavior and predict optimal geometries and/or performance under different environmental conditions. During KauaiEx, extensive oceanographic measurements were made and can be used to improve simulation fidelity. The thermistor-array data provided a sound-speed profile measurement every minute and could be assumed to be representative of oceanographic conditions between the source and receiver. Simulations for computing impulse responses were conducted for each profile and showed strong agreement with the measured responses. A model-generated impulse response was generated with each new sound speed profile measurement. The FSK signals were then convolved with the simulated channel impulse response, and demodulated by a virtual receiver. This was done for each of the 16 hydrophones in the vertical array for each sound-speedprofile measurement time step. The bit error percentage as a
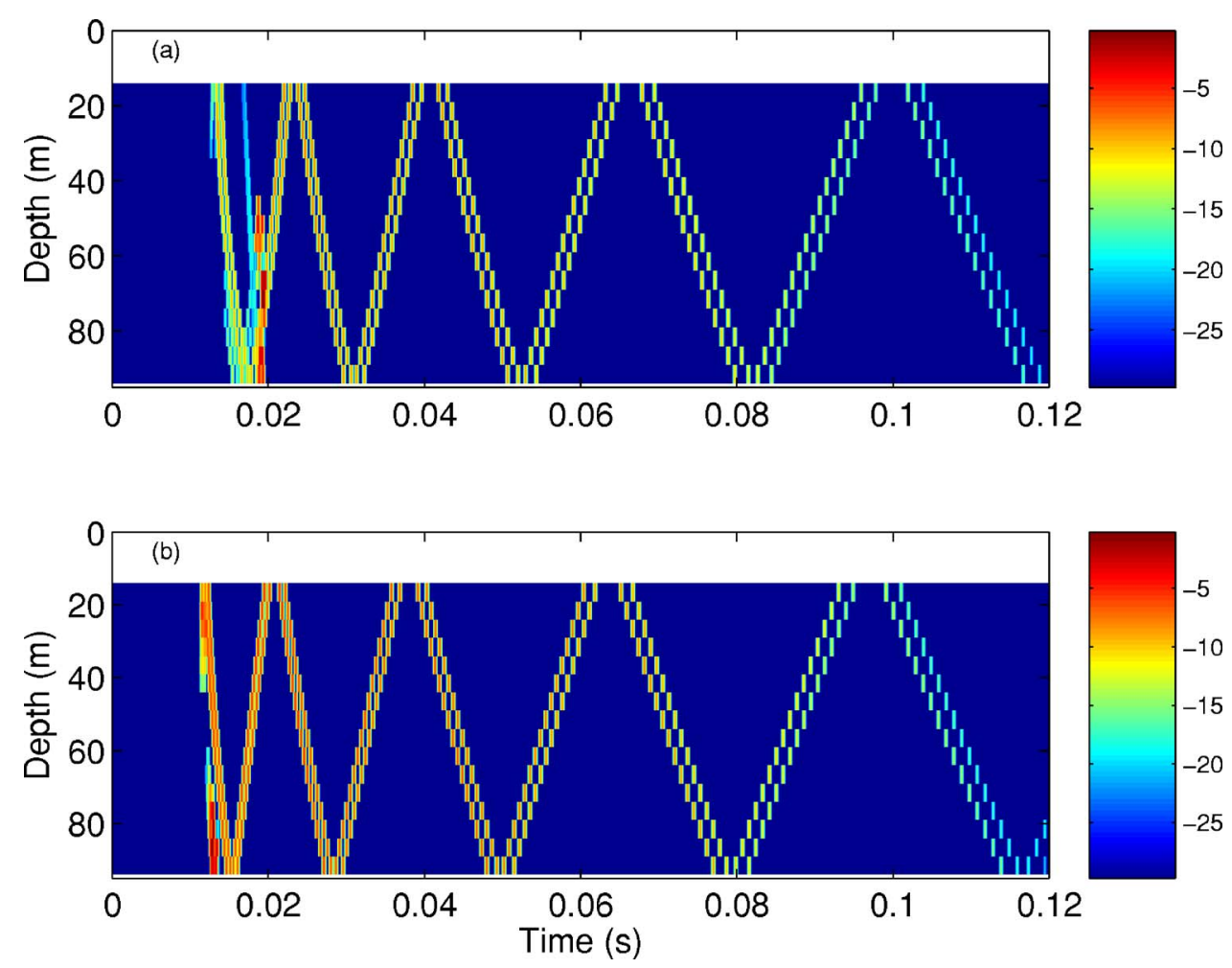

FIG. 12. (Color online) Simulated impulse responses from matched-filtered LFM transmissions at times with low and high bit errors. Each panel is on a relative scale of $30 \mathrm{~dB}$ with the highest overall peak set to $0 \mathrm{~dB}$. Panel (a) corresponds to period with low bit errors [measured impulse response at that time is shown in panel (a) in Fig. $10]$ and (b) to the period with high bit errors [corresponding to (b) of Fig. 10]. As with the measured impulse responses, the notable feature in (a) is the focusing of arrivals at the deepest hydrophones that produces a peak in time much larger than the other paths. 

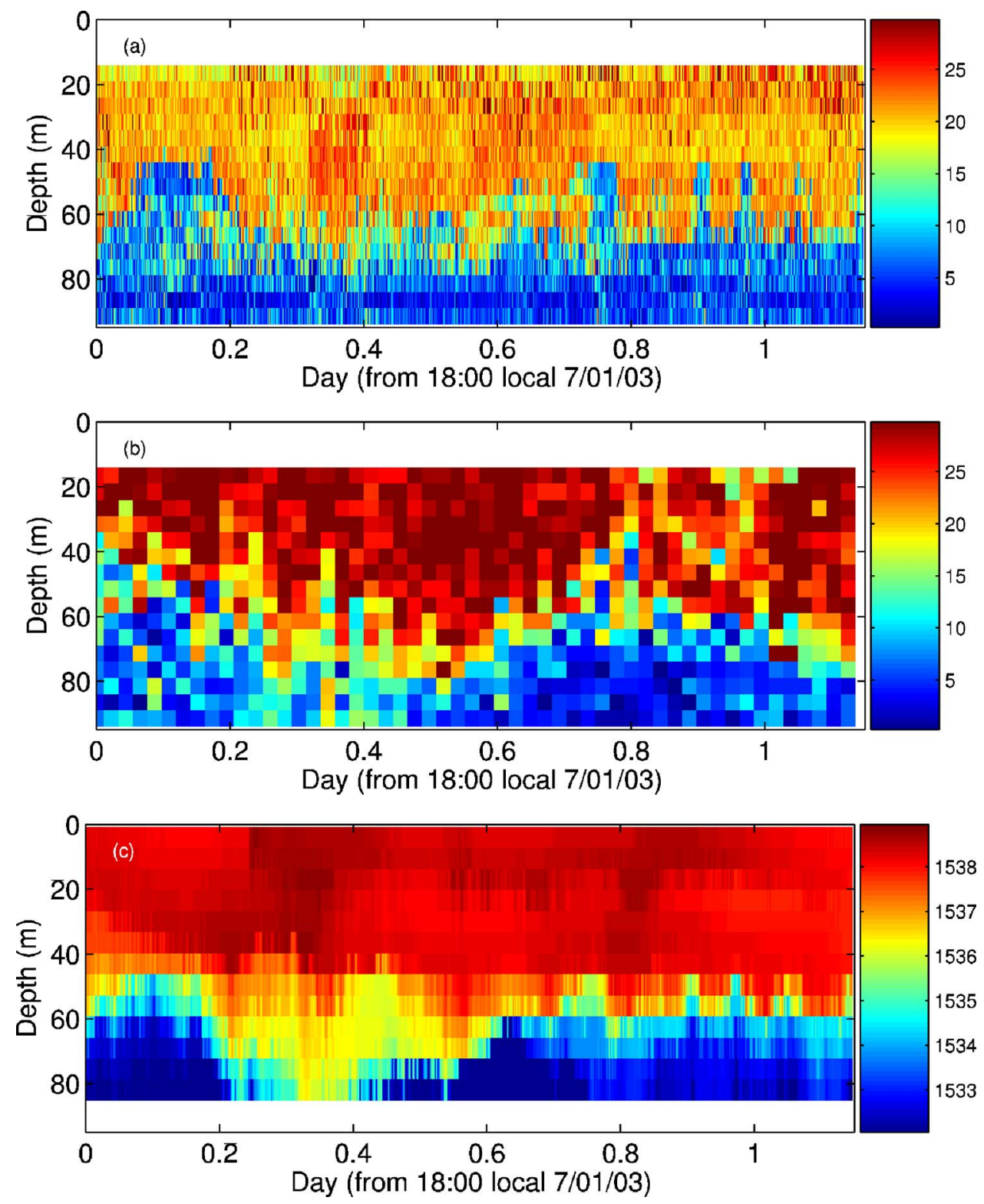

FIG. 13. (Color online) Panel (a) is the simulated percent bit errors at the 16 hydrophone depths over the 1 day experiment (percent error). Panel (b) is the same for the measured data (percent error). Panel (c) is the sound speed profile $(\mathrm{m} / \mathrm{s})$.

function of time is shown in Fig. 13 along with the measurements of the same quantity. Also shown, for comparison, is the sound-speed profile during the same period. From Fig. 13, several points can be made. First, the simulations and measurements are very similar in both time and space. Second, the upper and lower portions of the water column show very different bit errors in both the measurements and simulation. Third, both appear to track changes in the oceanography in a similar way. Last, it is interesting to note that the overall best performance was observed for the second deepest hydrophone (at about $86.5 \mathrm{~m}$ ) and this was duplicated in the simulation.

The simulator-computed fading characteristics were calculated in a similar manner to that discussed in Sec. III E. In Fig. 14, the envelope of the amplitude distributions for the same hydrophone (Sec. III E, second deepest) is shown for both high and low bit-error periods corresponding to when the thermocline was weak (mixed water column) and when it was strong, respectively. The distributions computed by the simulator and directly from experimental data show good agreement. A strong thermocline results in a shifted distribution toward higher amplitudes and an approximate Ricean curve fit. A more uniformly mixed water column results in lower amplitudes and an approximate Rayleigh curve fit.

Finally, the model-predicted, bit-error probabilities are compared with the theoretical performance curves for $M=4$ FSK signaling; the results are shown in Fig. 15. The points on the figure are color coded showing the upper 4 hydrophones in blue, the middle 8 hydrophones in green, and the deepest 4 hydrophones in red. As was the case for the measurements, the performance improves with depth as $\mathcal{E}_{b M} / N_{0 M}$ increases. As was the case for the measurements, at the low end of $\mathcal{E}_{b M} / N_{0 M}$ the errors are slightly worse than the Rayleigh-fading prediction. At the high $\mathcal{E}_{b M} / N_{0 M}$ end, the performance is much better than Rayleigh but not quite reaching the nonfading performance curve. It is important to 


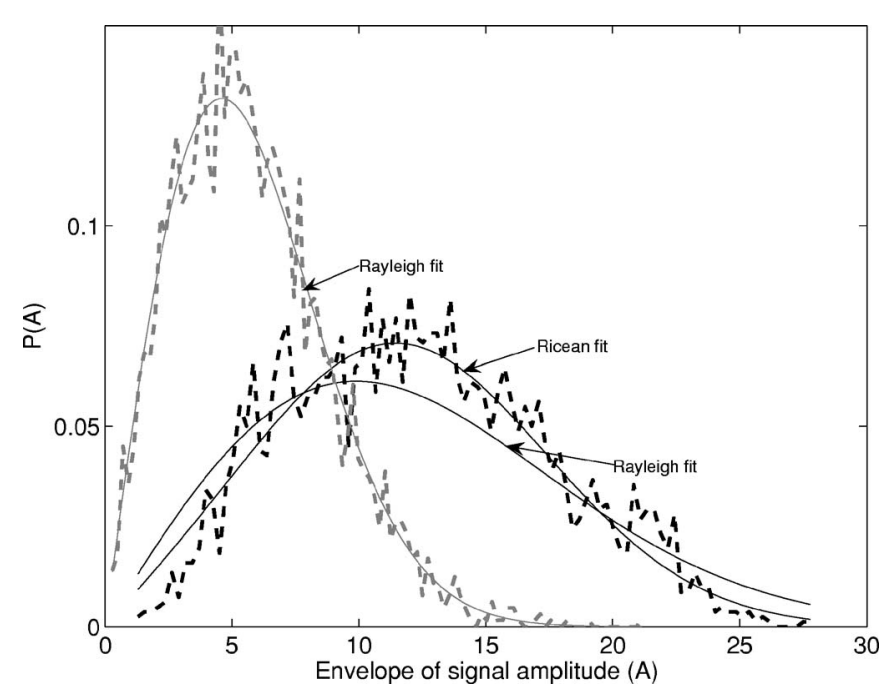

FIG. 14. Simulation: Envelopes of the amplitude distributions on a short time scale (data are taken from 3, $0.8 \mathrm{~s}$ transmissions) for just the deep hydrophone at $86.5 \mathrm{~m}$. During the period with the water column mixed, the errors increased and the amplitude distribution is shown by the gray dashed curve with the solid gray being the best fit to a Rayleigh distribution (higher BER). For the period with a strong thermocline, there were much fewer errors. The amplitude distribution is shown as a black dashed line with the best fit Rayleigh and Ricean curves as solid black lines (lower BER).

note here that this performance calculation was done without added noise. The performance is nearly the same as that measured indicating the background ambient noise has little to do with the performance in this regime. This was verified by adding background ambient noise (equal to that for the measurements) and there was no significant change in the simulated results shown in Fig. 15.

\section{DISCUSSION AND CONCLUSIONS}

The communication performance dependence on sourcereceiver geometry and oceanographic conditions have been described for FSK transmissions over distances of $3 \mathrm{~km}$ in the $8-13.2 \mathrm{kHz}$ band for an experimental site near Kauai, HI. Since the received signal level was well above the ambient noise level, the limiting factor in the performance was the multipath interference. Thus, key factors in the modem performance were the source/receiver geometry and the oceanography. Using measured sound-speed profiles, simulations were made to mimic the measured data collection over the 1 day experiment. Results showed a simulated performance very similar to that measured. This held true even in the absence of added ambient noise in the channel. This is somewhat counterintuitive but it implies that once the channel is no longer ambient-noise limited, increasing the source level has no impact on performance.

The greatest improvements in performance were achieved by changing the receiver depth (the transmitter depth was fixed). The communication signals from receivers in the middle of the water column showed fading consistent with a Rayleigh-fading model over much of the experiment duration. The shallowest hydrophones exhibited slightly worse fading characteristics and the deepest hydrophones were slightly better. During the most favorable periods when there was a strong thermocline, the deepest hydrophones ap-

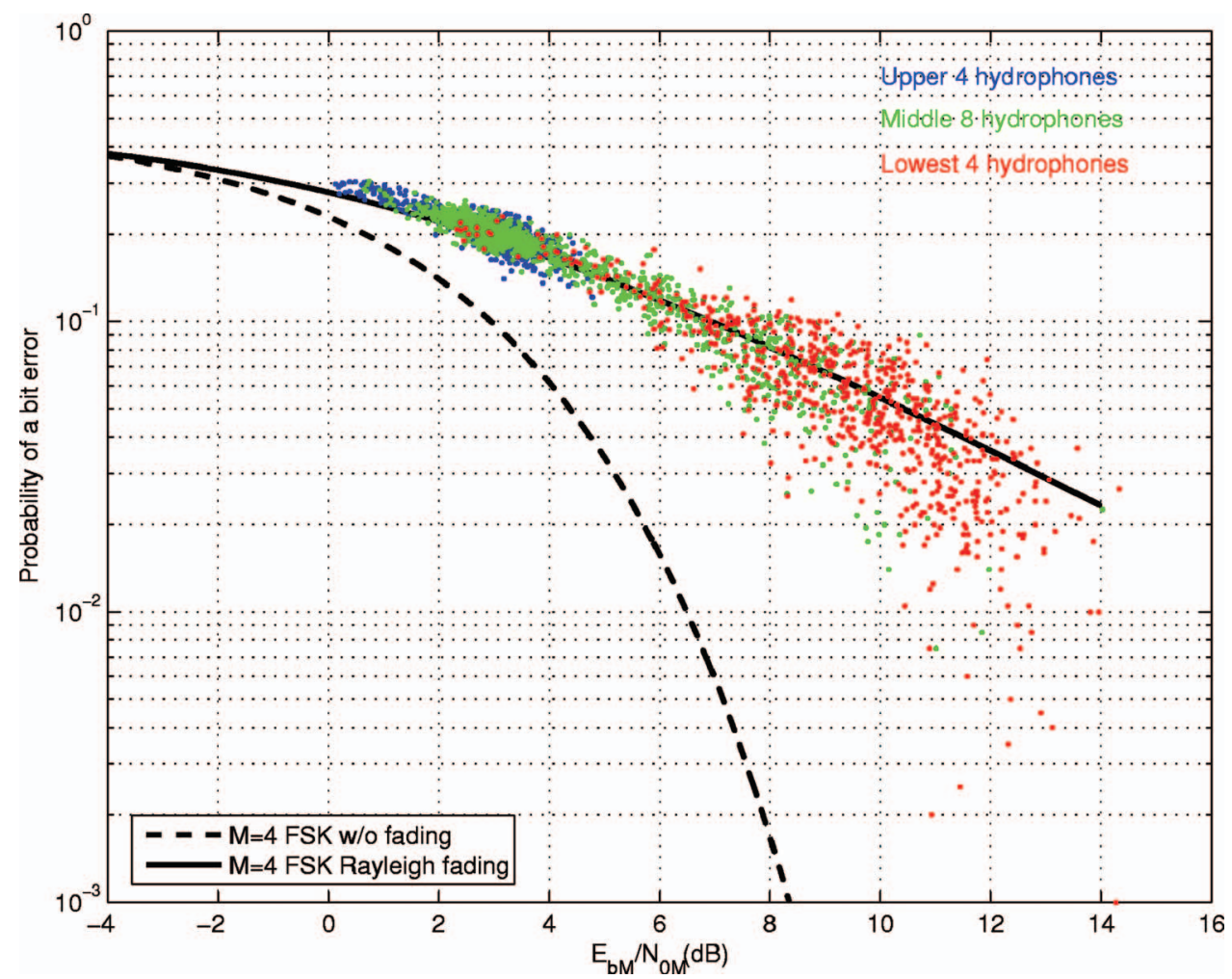

FIG. 15. Simulation: Probability of bit error for transmission during 1 day of KauaiEx vs $\mathcal{E}_{b M} / N_{0 M}$. The solid line is the theoretical performance for a Rayleigh fading channel and the dashed line is for a nonfading channel. The colored dots indicate depth within the water column. The blue dots are the shallowest hydrophones, the green dots are in the middle of the water column, and the red dots are the deepest hydrophones. This simulation was done without any ambient noise added. 
proached the characteristics of a nonfading channel. The thermocline varied significantly during the $24 \mathrm{~h}$ measurement period and there was a period when the thermocline nearly disappeared and the water column was entirely mixed (iso-speed). At this time, the deeper hydrophones lost their favorable conditions and error rates increased significantly.

Modeling was used to show how acoustic energy is trapped due to the thermocline giving rise to the observed favorable arrival structure in the lowest hydrophone depths. The favorable arrival structure is characterized by a very large amplitude arrival (or group of arrivals) that is not the earliest arrival(s). When the water column is mixed, the thermocline is gone and the lowest hydrophones show a similar impulse response to the shallower hydrophones and performance is similar.

Oceanographic conditions like these are common. Summer conditions often give rise to a strong thermocline, while winter conditions usually show more mixing resulting in isospeed profiles. These results, together with the modeling, show how the environment can play a significant role in underwater acoustic communications performance. In situations similar to those during KauaiEx, a strategy for optimizing performance might include avoiding transmission times when the water column is mixed and concentrating assets near the seabed as opposed to near the surface. While this is not a general rule since environmental conditions vary at different locations, the modeling results show how predictions can be made if sufficient environmental knowledge exists.

\section{ACKNOWLEDGMENTS}

This work was supported by the Office of Naval Research. We would like to express particular appreciation to the team from the Marine Physical Laboratory at the University of California, San Diego, William Hodgkiss, Jeff Skinner, and Dave Ensberg for the vertical array data used here. The authors also gratefully acknowledge the University of Delaware team, led by Mohsen Baidey, for the CTD and thermistor data used for this analysis. We would also like to thank Naval Research Enterprise Internship Program (NREIP) student Laura Meathe and SPAWARSYSCEN, San Diego employee Leo Ghazikhanian for their assistance with operating the Telesonar Testbed instrument. Additionally, we would like to acknowledge Joe Rice for the Telesonar Testbed concept and for his support during its development. The KauaiEx Group consists of: Michael B. Porter, Paul Hursky, Martin Siderius (HLS Research), Mohsen Badiey (University of Delaware), Jerald Caruthers (University Southern Mississippi), William S. Hodgkiss, Kaustubha Raghukumar (Scripps Institute of Oceanography), Daniel Rouseff, Warren Fox (University of Washington), Christian de Moustier, Brian Calder, Barbara J. Kraft (University of New Hampshire), Keyko McDonald (SPAWARSSC), Peter Stein, James K. Lewis, and Subramaniam Rajan (Scientific Solutions).

${ }^{1}$ M. B. Porter and the KauaiEx Group, "The Kauai experiment," in HighFrequency Ocean Acoustics (AIP, Melville, NY, 2004), pp. 307-321.

${ }^{2}$ M. Siderius, M. B. Porter, and the KauaiEx Group, "Impact of thermocline variability on underwater acoustic communications: Results from KauiEx," in High-Frequency Ocean Acoustics (AIP, Melville, NY, 2004), pp. 358-365.

${ }^{3}$ M. B. Porter, V. K. McDonald, P. A. Baxley, and J. A. Rice, "Signalex: Linking environmental acoustics with the signaling schemes," in Proceedings of MTS/IEEE Oceans00 (IEEE, New York, 2000), pp. 595-600.

${ }^{4}$ N. M. Carbone and W. S. Hodgkiss, "Effects of tidally driven temperature fluctuations on shallow-water acoustic communications at $18 \mathrm{kHz}$," IEEE J. Ocean. Eng. 25, 84-94 (2000)

${ }^{5}$ J. G. Proakis, Digital Communications, 3rd ed. (McGraw-Hill, New York, 1995).

${ }^{6}$ D. B. Kilfoyle and A. B. Baggeroer, "The state of the art in underwater acoustic telemetry," IEEE J. Ocean. Eng. 25, 4-27 (2000).

${ }^{7}$ J. Rice et al., "Evolution of seaweb underwater acoustic networking," in Proceedings of MTS/IEEE OCEANS'OO Conference (IEEE, New York, 2000), pp. 2007-2017.

${ }^{8}$ V. K. McDonald, P. Hursky, and the KauaiEx Group, "Telesonar testbed instrument provides a flexible platform for acoustic propagation and communication research in the $8-50 \mathrm{kHz}$ band," in High-Frequency Ocean Acoustics (AIP, Melville, NY, 2004), pp. 336-349.

${ }^{9}$ J. G. Proakis, "Coded modulation for digital communications over Rayleigh fading channels," IEEE J. Ocean. Eng. 16, 66-73 (1991).

${ }^{10}$ R. J. Urick, Principles of Underwater Sound (McGraw-Hill, New York, 1983).

${ }^{11}$ A. Zielinski, Y. H. Yoon, and L. Wu, "Performance analysis of digital acoustic communication in a shallow water channel," IEEE J. Ocean. Eng. 20, 293-299 (1995).

${ }^{12}$ C. Bjerrum-Niese, L. Bjorno, M. Pinto, and B. Quellec, "A simulation tool for high data-rate acoustic communication in a shallow-water time-varying channel," IEEE J. Ocean. Eng. 21, 143-149 (1996).

${ }^{13}$ M. B. Porter and H. P. Bucker, "Gaussian beam tracing for computing ocean acoustic fields," J. Acoust. Soc. Am. 82, 1349-1359 (1987).

${ }^{14}$ M. Siderius and M. B. Porter, "Modeling techniques for marine mammal risk assessment,” IEEE J. Ocean. Eng. 31, 49-60 (2006). 